\title{
Evaluation of Right Ventricular Function and Various Models of Delayed Enhancement with Cardiac Magnetic Resonance Imaging in Patients with Repaired Tetralogy of Fallot
}

\author{
Marzieh Motevalli ${ }^{1}$, Farzaneh Akhavan ${ }^{2}$, Ali Mohammadzadeh ${ }^{1}$, Zeinab Norouzi ${ }^{1}$, Navid \\ Abolfathzadeh ${ }^{3}$, Mohammad Ali Mohammadi Vajari ${ }^{4}$, Hamid Reza Pouraliakbar ${ }^{1}$, Kiara \\ Rezaei-Kalantari ${ }^{1}$, Sanaz Asadian ${ }^{1}$, Aminkasra Kiani ${ }^{5}$ and Ghazale Tefagh ${ }^{2,{ }^{*}}$ \\ ${ }^{1}$ Department of Radiology, Shahid Rajaie Cardiovascular, Medical and Research Center, Tehran, Iran \\ ${ }^{2}$ Student Scientific Research Center, Tehran University of Medical Sciences, Tehran, Iran \\ ${ }^{3}$ Department of Ophthalmology, Nikookari Eye Hospital, Tabriz University of Medical Sciences, Tabriz, Iran \\ ${ }^{4}$ Department of Radiology, Iran University of Medical Sciences, Tehran, Iran \\ ${ }^{5}$ Unified District School of Los Angeles, Los Angeles, California, United States \\ "Corresponding author: Student's Scientific Research Center, Tehran University of Medical Sciences, Tehran, Iran. Tel: +98-9132749107, Email: tefagh.gh@gmail.com
}

Received 2019 May 20; Revised 2020 February 10; Accepted 2020 February 16.

\begin{abstract}
Background: Tetralogy of Fallot (TOF) is considered as the most frequent cyanotic congenital heart disorder. Right ventricular (RV) dysfunction is possible to be observed in patients with repaired TOF. Delayed enhancement (DE) is one of the recommended findings for RV dysfunction.

Objectives: This study aimed at investigating the DE and its probable relationship with RV function through cardiac magnetic resonance (CMR).

Patients and Methods: In this cross-sectional study, the values of cardiovascular magnetic resonance of 110 symptomatic patients, who had repaired TOF for 35 years, were gathered. We compared cardiac function indices (CFI) in patients with and without DE.

Results: The patients had an average age of $21.93 \pm 6.94$ years (59.12\% were male and $40.94 \%$ were female). $93.66 \%$ of the subjects showed DE and $6.44 \%$ of them did not have DE. 78.61\% of the samples had DE of the right ventricular outflow tract (RVOT); whereas, 21.39\% showed DE of the other sites. The differences between mean regurgitation fraction, average RV end-diastolic volume (RVEDV), average RV end systolic volume (ESV), and average RV ejection fraction in DE positive and negative patients were statistically significant $($ P values $=0.01,0.04,0.04$, and 0.01, respectively.)

Conclusion: DE commonly occurs as a complication of surgery to repair TOF and could be used as a factor for impaired RV function and other complications. Using CMR imaging for follow up of these patients could lead to diagnosis of these complications.
\end{abstract}

Keywords: Tetralogy of Fallot (TOF), Delayed Enhancement (DE), Cardiac MRI (CMR), Tetralogy of Fallot Total Correction (TFTC)

\section{Background}

Tetralogy of Fallot (TOF) is considered as the most frequent cyanotic congenital heart disorder $(1,2)$. It has four characteristic components: large ventricular septal defect (VSD), right ventricular (RV) outflow tract obstruction, overriding aorta on the interventricular septum and right ventricle hypertrophy $(\mathrm{RVH})(3,4)$. The incidence of TOF is $0.3-0.5$ in every 1,000 live births $(5,6)$. Tetralogy of Fallot total correction (TFTC) has shown satisfactory and significant results for 40 - 50 years $(7,8)$.

Despite the good long-term prognosis in post-surgical status, these patients still have shorter life expectancies compared to the normal population. An increasing num- ber of adult patients who have had TOF repair surgery in their childhood now require regular follow up for their post-operative complications. The common post-surgical complications are pulmonic regurgitation (Figure1), residual pulmonary stenosis, residual VSD, right ventricular outflow tract aneurysm, right ventricular dysfunction, and arrhythmia $(6,7,9)$. Considering these major complications, regular follow-up after total surgical correction is necessary. Conventional imaging methods, including echocardiography and radionuclide studies are limited to assess these complications. Transthoracic echocardiography has the limitation of post-surgical scar tissue and thoracic deformities $(6,7)$. On the other hand, cardiac mag- 
netic resonance (CMR) has been proposed as the gold standard imaging modality for evaluation of right ventricle function $(6,10,11)$.
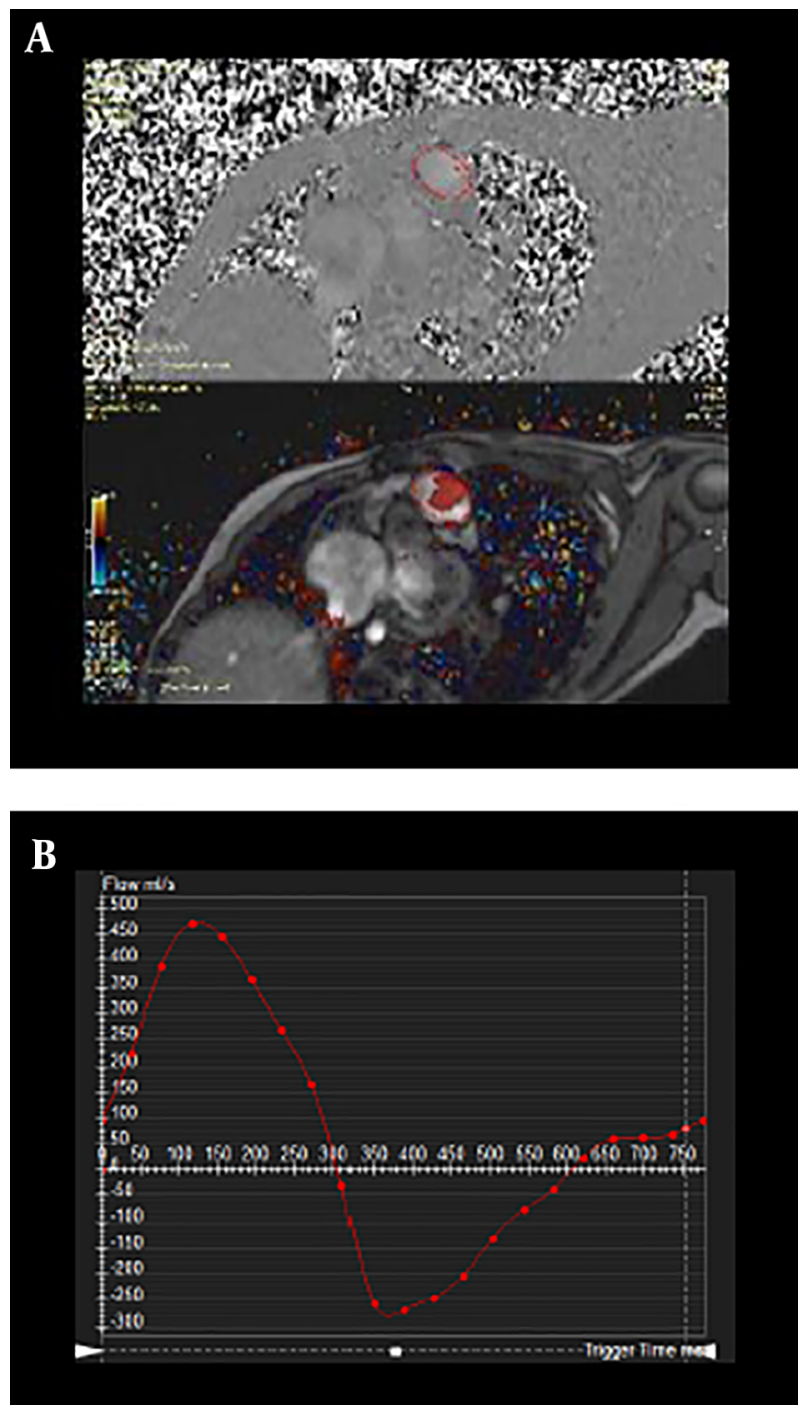

Figure 1. Flow measurement of a transverse slice at the level of main pulmonary artery, velocity encoded sequence on phase and magnitude (A). Flow profile of pulmonary artery; the area under the curve above the horizontal axis represents the antegrade flow and the area under the curve below the horizontal axis represents retrograde flow. The curve demonstrates severe pulmonary regurgitation (B).

Right ventricular dysfunction may occur as a serious complication in patients with complete repair of TOF with high morbidity and mortality rates (9). Different mechanisms have been proposed to contribute to global right ventricular (RV) dysfunction in patients with repaired TOF. This complication often occurs secondary to long term pulmonary valve regurgitation, which could result in right ventricular dilatation and increased risk of severe arrhyth- mias $(6,7)$. Delayed enhancement (DE) due to post-surgical scar tissue and fibrosis has been considered as an important finding in the development of right ventricular failure (12).

\section{Objectives}

The subject of cardiac MR in TOF patients in the Iranian society has not been explored up to now, considering the high incidence of TOF and the subsequent need for surgical treatment of these patients, this study was conducted to quantify delayed enhancement in right ventricular outflow tract (RVOT) using CMR after repairing surgery.

\section{Patients and Methods}

This is a descriptive analytic cross-sectional study performed on 110 consecutive symptomatic patients with repaired tetralogy of Fallot referred to Shaheed Rajaie hospital for clinical and radiological evaluation with CMR with the history of correction surgery during the past 35 years. This study evaluates clinical and CMR data from these patients. Inclusion criterion was symptomatic TOF in TFTC patients. Exclusion criteria were TOF patients with shunt surgery or inadequate clinical information for CMR. After providing full information about the methods and aims of the study, informative consent from each patient was obtained upon entry into the study. The local ethics committee confirmed the protocol of this research and it was conducted in accordance with the Helsinki declaration.

The samples were selected using the census method. All patients with sufficient inclusion criteria were enrolled in the study. The clinical and CMR data were collected from the patient registry information. The sample size was calculated by using the "one trait in the population" formula. The first type of error and the incidence of DE were assumed $5 \%$ and $70 \%$, respectively (13).

\subsection{CMR Imaging and Images Review}

In this study, cardiac MR was used to estimate ventricular volumes (Figure 2) and function and also to quantify the flow vessel over the entire cardiac valves and large vessels. CMR imaging was performed on 1.5 Tesla MR system (Magnetom Avanto, Siemens Medical Systems, Germany).

10 - 14 contiguous short- axis slices with a thickness of $8 \mathrm{~mm}$ and a gap of $25 \%$ were provided by steady-state free precession (SSFP) cine imaging covering the heart from apex to base. The software package (Argus, Siemens, Germany) was employed for measuring the ventricular enddiastolic volume (VEDV) and end-systolic volume. The data were adjusted for body size. 

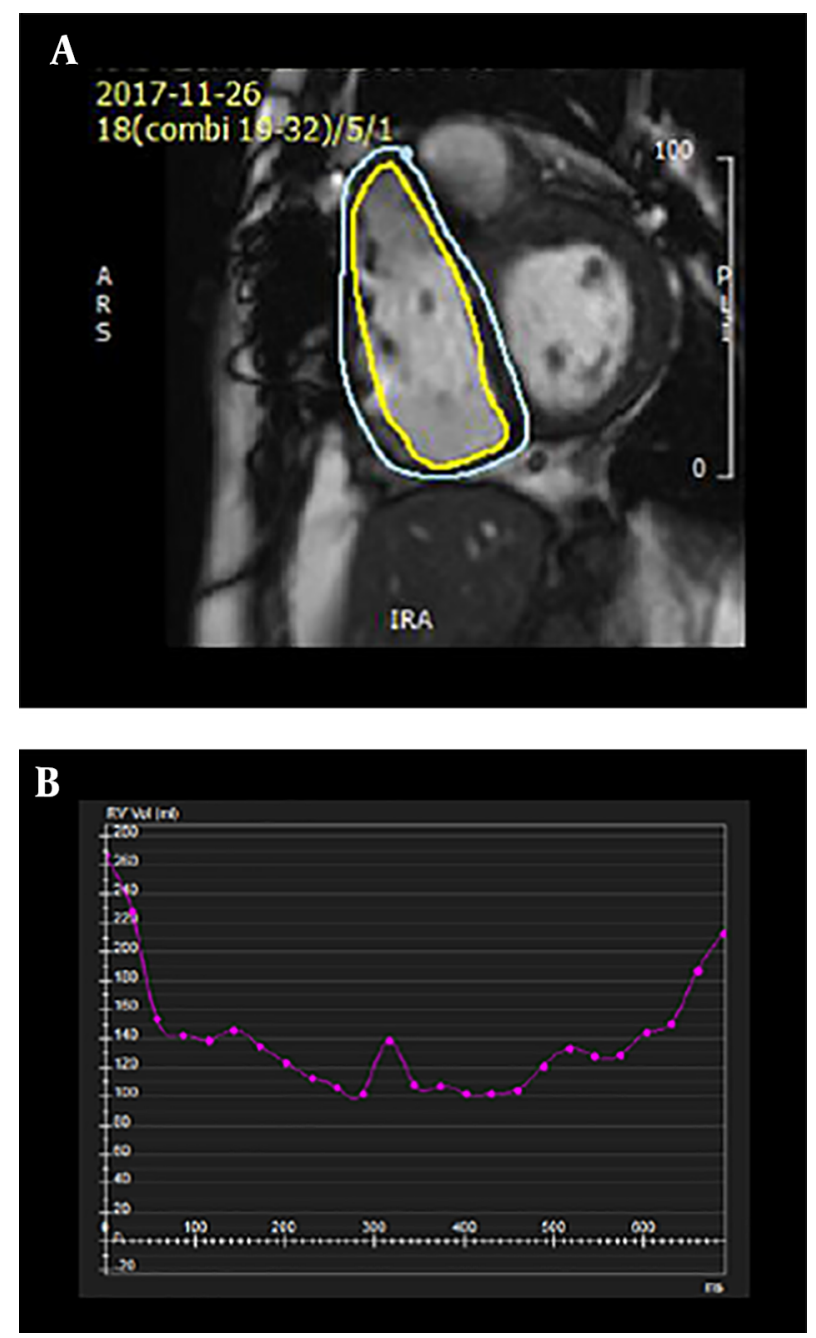

Figure 2. Severe right ventricular (RV) enlargement in tetralogy of Fallot total correction (TFTC). Severe increased RV volume in diastole and systole post TFTC (right ventricular end-diastolic volume $[\mathrm{RVEDV}]=270 \mathrm{~mL}$, right ventricular end systolic volume [RVESV] = $100 \mathrm{~mL}$ ) (A). Time -volume curve of RV during cardiac cycles (B).

An additional cine imaging was used for RVOT area measurements and presented in the longitudinal axis of RVOT. The highest anterior-posterior diameter was obtained perpendicular to the long axis of RVOT at enddiastole. RVOT was considered aneurysmal if the diameter was more than $40 \mathrm{~mm}$.

The regurgitation fraction was determined as regurgitation volume divided by pulmonary forward volume via pulmonary trunk velocity map. Velocity mapping was performed using a velocity encoding range of $150 \mathrm{~cm} / \mathrm{s}$. Velocity encoding was adjusted upon aliasing. The adjusted RVEF is defined as the net pulmonary forward volume divided by the right ventricular EDV (RVEDV). The net for- ward flow was measured by the difference between volumes of forward flow and pulmonary regurgitate.

MR imaging was carried out through single shot phase sensitive inversion-recovery technique and 0.2 millimol/kg intravenous gadoteric acid (Dotarem, Guebert, France) with 2.8 - $3 \mathrm{cc} / \mathrm{sec}$ flow injection rate 10 - $15 \mathrm{~min}$ following MR angiography of the pulmonary vascular tree. The following characteristics were introduced for a standard imaging: a field of view of $400 \times 400 \mathrm{~mm}$, a thickness of $1 \mathrm{~mm}$ with no gap, a [256 $\times 256]$ matrix and flip angle of 15.20 - 30 sections were prepared through a 13 to 25 second breath hold, which was associated with the size and rate of heart in patient. An inversion time of $300 \mathrm{~ms}$ was set to achieve appropriate nulling of the normal myocardium through real-time imaging.

Two observers agreed on the presence or absence of delayed enhancement of the heart. They looked for DE in the free wall of the RV outflow tract, the interventricular septum and other sites of heart (Figure 3). The patients with and without DE were compared for cardiac function indices.

All statistical analyses were performed with SPSS Statistics for Windows, version 15.0 (SPSS Inc., Chicago, Ill., USA). We used frequency and ratio and also the mean and standard deviation for categorical and quantitative continuous variables, respectively. We compared proportions and means were performed using chi- 2 test and $t$-test, respectively. Relationship between quantitative variables was determined using Pearson coefficient. P values of smaller than 0.05 were regarded as significant.

\section{Results}

In this study, 110 patients (65 [59.12\%] male and 45 [40.94\%]) female with the mean age of $21.93 \pm 6.94$ years were included. Of the studied patients, a seven-year old patient and a 48-year old subject were found as the youngest and the oldest patients. The mean time interval between initial surgery and CMR study was approximately 14.61 \pm 6.01 years ( 1 - 35 years). 103 subjects (93.66\%) had DE, whereas seven subjects (6.44\%) did not. The data regarding frequency and location of DE are shown in Table 1.

Delayed enhancement was seen in $95.47 \%$ (62 patients) and $91.16 \%$ (41 patients) of male and female patients, respectively. This yielded no significant gender difference ( $P$ $=0.17$ ). The mean age for patients with and without DE were $21.92 \pm 7.18$ and $19.75 \pm 3.68$ years, respectively, which was not significant $(\mathrm{P}=0.42)$.

In our study, RVOT aneurysm was seen in 99 patients (90\%) and pulmonary regurgitation was found in 95 patients (86.43\%). Seventy-four patients (67.37\%) showed no 

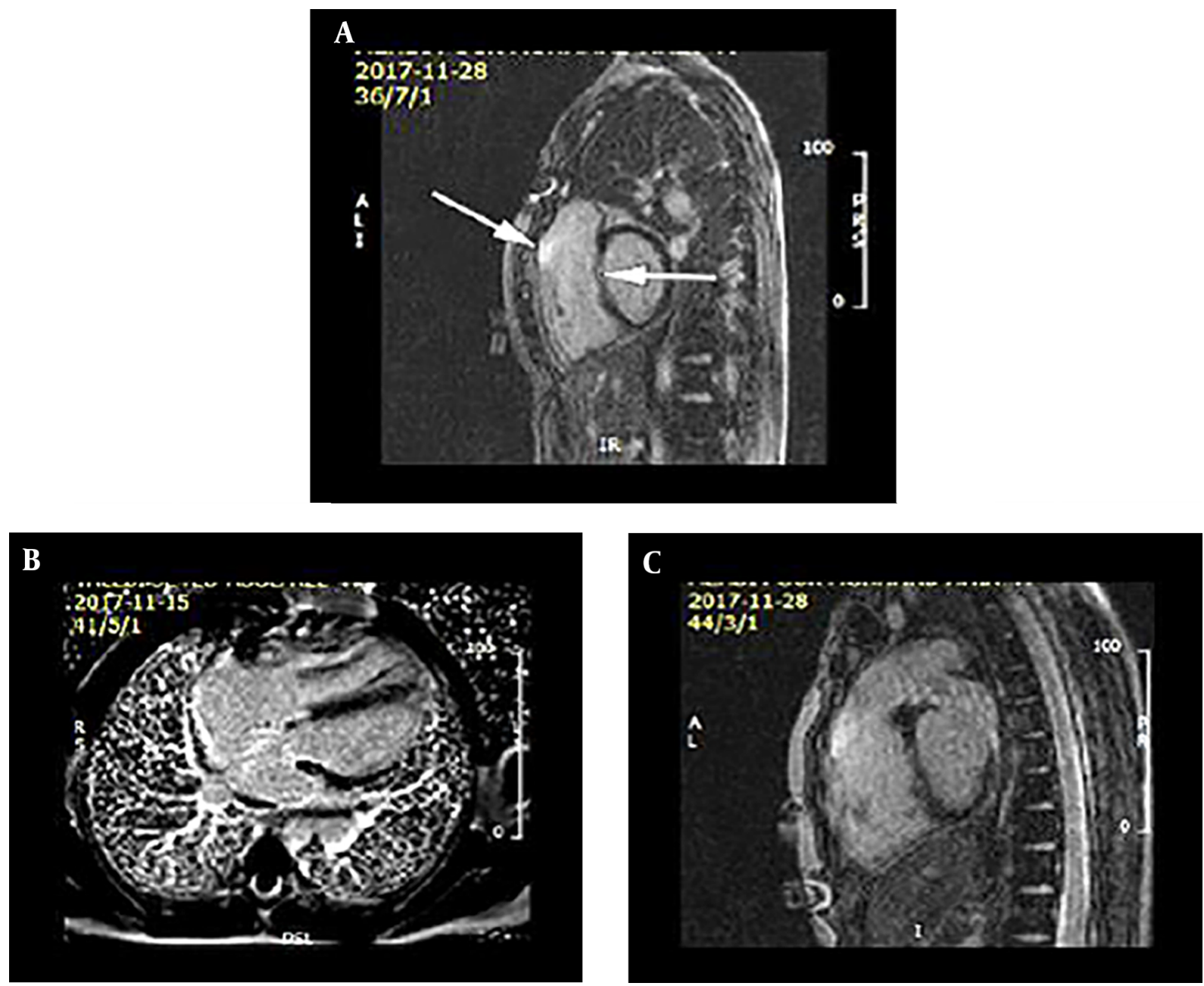

Figure 3. Phase sensitive inversion recovery sequence post gadolinium in sagittal $(\mathrm{A}, \mathrm{C})$ and four chamber axial views (B) demonstrate delayed enhancement in right ventricular outflow tract (RVOT) and ventricular septal defect (VSD) repair site in tetralogy of Fallot total correction (TFTC) represent scar in surgical site.

evidence of pulmonary stenosis while the remaining 36 patients (33.72\%) had pulmonary stenosis. Residual VSD was positive in 28 patients (25.56\%) leaving the remaining 82 patients (74.58\%) with no residual VSD. Residual VSD was usually small size in these 28 patients.

The relation between right ventricular function indices and DE is shown in Table 2. The content of this table indicates a significant relationship between DE with factors affecting right ventricular function, such as regurgitation fraction, RVEDV, right ventricular end systolic volume (RVESV), and right ventricular ejection fraction (RV EF).

The relation between DE and mean time after surgery was also investigated. The results showed that the mean duration in patients without DE was $15.68 \pm 4.72$ years and in patients with DE was $14.57 \pm 6.29$ years. Comparison of these values showed no significant difference.

\section{Discussion}

According to the results, DE can be found in patients with repaired TOF as well as $93.66 \%$ of symptomatic patients after surgery showed DE. Delayed enhancement could be an indication of ventricular scarring or fibrosis in patients without ischemic heart disease. Right ventricular DE in TOF patients after surgical repair was seen in 83 $\%$ and $92 \%$ of patients in studies conducted by Oosterhof et al. and Lu et al., respectively $(12,13)$ which are similar to our study. A study of children with congenital heart disease (including not only TOF) carried out by Harris et al. in 2007 found DE rates of $91 \%$ after surgical repair of the anomalies. In this study, DE has been seen in 3\% of children without any previous surgery (14). Babu-Narayan claimed a right ventricular DE rate at surgical site of $99 \%$ in his study, 


\begin{tabular}{|lcc|}
\hline Table 1. Frequency and Location of Delayed Enhancement & \\
\hline Location of delayed enhancement & Number of patient & Percent \% \\
\hline Variable & 81 & 73.61 \\
\hline IVS & 1 & 0.90 \\
\hline RVOT + right side of the septum & 4 & 3.63 \\
\hline Right ventricular septal junction & 2 & 1.77 \\
\hline RVOT + IVS at the site of VSD patch & 5 & 4.53 \\
\hline RVOT + both RV septal junction & 1 & 0.87 \\
\hline $\begin{array}{l}\text { RVOT + RV apical + LV apicolateral } \\
\text { segment }\end{array}$ & 1 & 0.89 \\
\hline RVOT + Inferior RV septal junction & 2 & 1.81 \\
\hline Inferior RV septal free wall junction & 2 & 1.78 \\
\hline RVOT + anteroseptal segment & 2 & 1.73 \\
\hline Basal septal at the side of VSD patch & 1 & 0.92 \\
\hline RVOT + transmural apical segment & 1 & 0.97 \\
\hline Non-delayed enhancement & 7 & 6.44 \\
\hline Total & 110 & 100 \\
\hline Abbreviations: IVS, interventricur & & \\
\hline
\end{tabular}

Abbreviations: IVS, interventricular septum; LV, left ventricle; RV, right ventricle; RVOT, right ventricular outflow tract; VSD, ventricular septal defect

\begin{tabular}{lccc}
\hline \multicolumn{4}{l}{ Table 2. Relation Between Right Ventricular Function Indices and $\mathrm{DE}^{\mathrm{a}}$} \\
\hline Variable & DE positive & DE negative & P value \\
\hline Regurgitation fraction & $13.91 \pm 38.12$ & $16.72 \pm 22.36$ & 0.010 \\
RV end diastolic volume & $91.88 \pm 258.26$ & $38.25 \pm 185.51$ & 0.047 \\
\hline RV end systolic volume & $71.16 \pm 162.87$ & $35.96 \pm 103.31$ & 0.045 \\
RV ejection fraction & $7.79 \pm 38.23$ & $9.14 \pm 44.89$ & 0.048 \\
\hline LV ejection fraction & $41.41 \pm 55.25$ & $4.30 \pm 60.12$ & 0.421 \\
\hline LV end diastolic volume & $38.78 \pm 124.93$ & $20.77 \pm 129.15$ & 0.801 \\
\hline
\end{tabular}

Abbreviations: DE, delayed enhancement; LV, left ventricle; RV, right ventricle SD, standard deviation

${ }^{\mathrm{a}}$ Values are expressed as mean $\pm \mathrm{SD}$.

which is similar to our result of $93.66 \%$ (15). They observed left ventricular DE in $5 \%$ of the patients, which was not seen in our study. The most common sites of DE in our study were RVOT (73.61\%), RVOT with other regions (21.39\%) and in other areas except RVOT (5\%). Incidence of DE in RVOT has been $70 \%$ in the study performed by Oosterhof et al. (12).

Most of our patients (86.4\%) had pulmonary regurgitation and $36.6 \%$ of our patients had pulmonary stenosis, while in other studies, pulmonary stenosis has been reported in $10 \%-15 \%$ of the subjects (10). Pulmonary stenosis has usually been seen in the proximal part of RVOT up to the distal branches, which sometimes necessitates reoperation. This difference could possibly be explained by differences in surgical techniques. These findings indicate that despite repairing surgery in patients with TOF, these post-operative abnormalities in the majority of patients ultimately would lead to RV dysfunction.

We have shown that the presence of DE has a meaningful relationship with impaired right ventricular function, while indices of left ventricular function, such as left ventricular end diastolic volume (LV EDV) and left ventricular ejection fraction (LV EF) are not influenced. It seems that left ventricular function in TOF patients with reconstructive surgery is not affected by DE. This is in par with results of previous studies. Significant relationship was seen between DE and RV dysfunction (end-systolic RV volume and RVEF) in a study conducted by Babu-Narayan et al. (15). A significant relationship between DE and RV EDV was also established in the study conducted by Lu et al. (13).

RVOT diameter and RV end-diastolic volume were increased in patients with positive DE in the study carried out by Oosterhof et al., and they both had a significant inverse relationship with RV ejection fraction (12). Wald and colleagues analyzed MRI data from 256 patients with repaired TOF and scored them based on the number of enhanced Voxels in each area. They showed that a higher DE score is associated with lower right ventricular EF (9). A significant reverse correlation between DE and RVEF was found in our study too.

Harris and colleagues evaluated DE in MRI of 73 children with congenital heart disease after surgical correction and reported significant preserved RV ejection fraction $(61 \% \pm 9 \%)$ despite the presence of DE. This difference could possibly be due to differences between pediatric and adult populations (14). In their study, DE was present in structures not directly involved in reconstructive surgery, such as ascending aorta, which could be a result of inflammation of the aortic wall. In our study, there was no DE seen in this location.

We observed no statistically significant relationship between patients' age and gender and the incidence of DE, while Babu-Narayan et al. reported higher levels of right ventricular DE in older patients (15). Our results showed mild tricuspid regurgitation in 21 patients (19\%), while Norton and colleagues found moderate to severe tricuspid insufficiency in about $10 \%$ of the patients. They concluded that tricuspid insufficiency could develop due to annular dilatation of the valve subsequent to progressive right ventricular dilation (10). Marcelo et al. found that both ventricles show abnormal high fibrosis signal after TOF repair, in contrary to our results, in their study on 30 children with a history of TOF repair, Marcelo et al. claimed that both ventricles have similar abnormal high fibrosis signal (16). This can partially be explained by considering the differences in the surgical procedures between the two studies as well as the anatomical variations in TOF patients (17), also the small sample size of their study could be another explana- 
tion for the difference seen in their results.

In conclusion, in this study we aimed to explore the subject of cardiac MR in TOF patients in the Iranian society, which has not been explored up to now and also to find the possible differences between our population and other previously studied populations. The results of our study in par with previous studies have shown that DE, which can affect the right ventricle function, is a common finding following TOF repair surgery. Therefore, CMR imaging is the modality of choice for follow up of these patients after repairing surgery.

\section{Footnotes}

Authors' Contributions: Study concept and design: Marzieh Motevalli and Hamid Reza Pouraliakbar, acquisition of data: Navid Abolfathzadeh, Farzaneh Akhavan, and Zeinab Norouzi; analysis and interpretation of data: Navid Abolfathzadeh and Farzaneh Akhavan; drafting of the manuscript: Ghazale Tefagh, Mohammad Ali Mohammadi Vajari, and Marzieh Motevalli; critical revision of the manuscript for important intellectual content: Ghazale Tefagh, Mohammad Ali Mohammadi Vajari, Marzieh Motevalli, Hamid Reza Pouraliakbar, Kiara Rezaei-Kalantari, and Sanaz Asadian; statistical analysis: Navid Abolfathzadeh, Farzaneh Akhavan, and Zeinab Norouzi; administrative, technical, and material support: Marzieh Motevalli, Hamid Reza Pouraliakbar, Kiara Rezaei-Kalantari, Sanaz Asadian, Ali Mohammadzadeh, and Aminkasra Kiani; study supervision: Marzieh Motevalli

Conflict of Interests: Authors have no conflict of interests.

Ethical Approval: The local ethics committee confirmed the protocol of this research and it was conducted in accordance with the Helsinki declaration.

Funding/Support: The authors received no financial support for the research, authorship, and/or publication of this article. This research did not receive any specific grant.

Informed Consent: This is a retrospective study involving collection and analysis of data. However, informative consent was obtained from each patient upon entry into the study.

\section{References}

1. Anderson RH, Weinberg PM. The clinical anatomy of tetralogy of Fallot. Cardiol Young. 2005;15 Suppl 1:38-47. doi: 10.1017/s1047951105001010. [PubMed: 15934690].

2. Al Habib HF, Jacobs JP, Mavroudis C, Tchervenkov CI, O'Brien SM, Mohammadi S, et al. Contemporary patterns of management of tetralogy of Fallot: Data from the Society of Thoracic Surgeons
Database. Ann Thorac Surg. 2010;90(3):813-9. discussion 819-20. doi: 10.1016/j.athoracsur.2010.03.110. [PubMed: 20732501].

3. Kanter KR, Kogon BE, Kirshbom PM, Carlock PR. Symptomatic neonatal tetralogy of Fallot: Repair or shunt? Ann Thorac Surg. 2010;89(3):858-63. doi: 10.1016/j.athoracsur.2009.12.060. [PubMed: 20172143].

4. Aboulhosn J, Child JS. Management after childhood repair of tetralogy of Fallot. Curr Treat Options Cardiovasc Med. 2006;8(6):474-83. doi: 10.1007/s11936-006-0036-4. [PubMed: 17078912].

5. Starr JP. Tetralogy of fallot: Yesterday and today. World J Surg. 2010;34(4):658-68. doi: 10.1007/s00268-009-0296-8. [PubMed 20091166].

6. van Straten A, Vliegen HW, Hazekamp MG, de Roos A. Right ventricular function late after total repair of tetralogy of Fallot. Eur Radiol. 2005;15(4):702-7. doi: 10.1007/s00330-004-2574-z. [PubMed: 15726380].

7. Oosterhof T, Mulder BJ, Vliegen HW, de Roos A. Cardiovascular magnetic resonance in the follow-up of patients with corrected tetralogy of Fallot: A review. Am Heart J. 2006;151(2):265-72. doi: 10.1016/j.ahj.2005.03.058. [PubMed: 16442887].

8. Baharestani B, Rostami M, Omrani GR, Yousefnia M, Raeisi K, Givtaj N, et al. Evaluation of pulmonary regurgitation following tetralogy of fallot repair. Iran Heart J. 2010;11(2):14-24.

9. Wald RM, Haber I, Wald R, Valente AM, Powell AJ, Geva T. Effects of regional dysfunction and late gadolinium enhancement on global right ventricular function and exercise capacity in patients with repaired tetralogy of Fallot. Circulation. 2009;119(10):1370-7. doi 10.1161/CIRCULATIONAHA.108.816546. [PubMed: 19255342]. [PubMed Central: PMC2764308].

10. Norton KI, Tong C, Glass RB, Nielsen JC. Cardiac MR imaging assessment following tetralogy of Fallot repair. Radiographics. 2006;26(1):197-211. doi: 10.1148/rg.261055064. [PubMed:16418252].

11. Powell AJ, Nielsen JC. Cardiovascular MRI applications in congenital heart disease. Indian J Radiol Imaging. 2007;17(2):86. doi: 10.4103/09713026.33618.

12. Oosterhof T, Mulder BJ, Vliegen HW, de Roos A. Corrected tetralogy of Fallot: Delayed enhancement in right ventricular outflow tract. $R a$ diology. 2005;237(3):868-71. doi: 10.1148/radiol.2373041324. [PubMed: 16304108]

13. Lu JC, Cotts TB, Agarwal PP, Attili AK, Dorfman AL. Relation of right ventricular dilation, age of repair, and restrictive right ventricular physiology with patient-reported quality of life in adolescents and adults with repaired tetralogy of Fallot. Am J Cardiol. 2010;106(12):1798-802 doi: 10.1016/j.amjcard.2010.08.021. [PubMed: 21126623].

14. Harris MA, Johnson TR, Weinberg PM, Fogel MA. Delayedenhancement cardiovascular magnetic resonance identifies fibrous tissue in children after surgery for congenital heart disease. J Thorac Cardiovasc Surg. 2007;133(3):676-81. doi: 10.1016/j.jtcvs.2006.10.057. [PubMed: 17320564].

15. Babu-Narayan SV, Kilner PJ, Li W, Moon JC, Goktekin O, Davlouros PA et al. Ventricular fibrosis suggested by cardiovascular magnetic resonance in adults with repaired tetralogy of Fallot and its relationship to adverse markers of clinical outcome. Circulation. 2006;113(3):40513. doi: 10.1161/CIRCULATIONAHA.105.548727. [PubMed: 16432072].

16. Kozak MF, Redington A, Yoo SJ, Seed M, Greiser A, Grosse-Wortmann L. Diffuse myocardial fibrosis following tetralogy of Fallot repair: A T1 mapping cardiac magnetic resonance study. Pediatr Radiol. 2014;44(4):403-9. doi: 10.1007/s00247-013-2840-9. [PubMed: 24419492].

17. Ordovas KG, Muzzarelli S, Hope MD, Naeger DM, Karl T, Reddy GP, et al. Cardiovascular MR imaging after surgical correction of tetralogy of Fallot: Approach based on understanding of surgical procedures. Radiographics. 2013;33(4):1037-52. doi: 10.1148/rg.334115084. [PubMed: 23842971]. 